\title{
A technical update of high intensity focused ultrasound ablation for prostate cancer and benign prostatic hyperplasia
}

High-intensity focused ultrasound (HIFU) treatment for prostate cancer and benign prostatic hyperplasia (BPH) was utilized worldwide owing to benefits of non-invasive treatment, fast recovery time, and low rate of complications. There are many approaches of HIFU for BPH and prostate cancer under the guidance of real-time magnetic resonance imaging or ultrasound and the location of treatment transducer inside the rectum or urethra. In this article, we aimed to introduce these approaches for clinicians to consider as an alternative treatment option.

\section{KEYWORDS: Benign prostatic hyperplasia a prostate cancer a high-intensity focused ultrasound a non-invasive treatment}

\section{Introduction}

The prostate gland is the one of the most important organs belonging to men's reproductive system that generates the ejaculatory fluid for carrying sperm. Prostate gland is below the bladder and encircles the urethra, which is adjacent to vesicles and rectum. With aging, the prostate will become enlarged with unclear causes, generating the most common disorder known as benign prostatic hyperplasia (BPH). Even though, $\mathrm{BPH}$ is non-cancerous, disease can produce dissimilar urinary symptoms, such as frequent urge to urinate, urinary retention and nocturia. Meanwhile, prostate cancer is one of the most common men's cancer disease affected the entire prostate called diffuse prostate cancer or just a small part of gland called focal prostate cancer. Prostate cancer's symptoms, unclear in the early stage, are urinary disorder, hematuria, pelvic pain, leg numbness, and erectile dysfunction. The seriousness of the prostate cancer is relied on a combination of the data from the screening tests such as prostatespecific antigen (PSA), rectal examination, and biopsy. If diagnosed early, prostate cancer is possibly curable [1-4].

High-intensity focused ultrasound (HIFU) under the real-time guidance of magnetic resonance imaging or ultrasound to precisely minimize the damage of non-targeted healthy tissue is a non-invasive method, low-morbidity therapeutic option, radiation-free technique to eradicate ailment prostate tissue. The clinicians manage a beam path of ultrasonic energy to a chose volume of prostate lesion. The energy will heat and eliminate the targeted tissue at the focal spot by elevating the tissue's temperature up to the threshold of protein denaturation. This process is repeated until the entire selected volume or the entire gland is destroyed. The patient can achieve a short recovery time and a fast return to normal lifes activities with a preservation of significant surrounding organs and sexual function. Food and Drug Administration has recently approved HIFU for curing prostate diseases: prostate cancer and benign prostatic hyperplasia (BPH) [1-4]. There are some HIFU machines worldwide used which are divided into two groups with ultrasound guidance (Sonablate, Ablatherm, and Focal one) and with magnetic resonance imaging (MRI) guidance (Tulsa-Pro and Exablate). In this article, we aimed to introduce these approaches for prostate cancer and $\mathrm{BPH}$.

\section{- Ultrasound guided transrectal HIFU}

Ultrasound-guided transrectal HIFU treatment was exploited the transrectal HIFU probe with low-energy ultrasound of $3-4 \mathrm{MHz}$ for both imaging the prostate and emitting focused ultrasonic energy (FIGURE 1). Computer controlled positioning system manages the probe and sonication beam path to a specific prostate's lesion. Under computer control, treatment cells were further overlapped laterally and longitudinally to guarantee that the complete targeted area was 
comprehensively necrotic. Prior to the treatment, patient was adopted an enema and located in the lithotomy position with spinal anaesthesia. Foley urethral catheter was used to insert during the positioning phase of the treatment so as to expedite the bladder neck, prostatic urethra and seminal colliculus. Normal saline of 50-100 mL was dropped into the bladder to clarify the observation of bladder neck and urethra.

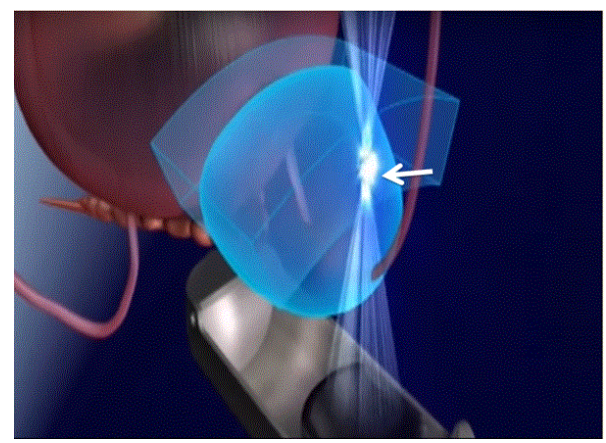

Figure 1. Ultrasound-guided HIFU to the targeted lesion (white arrow).

A condom was used to place over the transducer. The probe was put into the rectum and the covering sheath was dilated with 10-50 $\mathrm{mL}$ of degassed water to avoid sonicating through small air and maintain that the rectal temperature of $18-20^{\circ} \mathrm{C}$. The probe was wrapped consistently with ultrasonic coupling agent and then embedded into the rectum. Real-time ultrasound was carried out to map the treatment area and surrounding regions. The, the probe was established with the multiarticulated arm in fixed position. The targeted lesion was in the sonication length of $3-4 \mathrm{~cm}$. Prostatic tissue encircling urethra was selected as ablation regions. Longitudinal targeted section should include the total length of prostatic urethra tissues while 7-15 sections were selected in a transversal plane. It is noted that a urethral catheter was used during the imaging phase and removed when therapy was initiated. When the patient position was shifted due to movements or the reflective index in the targeted lesion was abnormally urged, the ablation was contemporarily stopped, the probe need to be repositioned before continuing treatment [5-8].

\section{- MRI guided HIFU}

Multiparametric MRI is presently the best imaging modality for the diagnosis of prostate diseases. In addition, MRI can be exploited to guide squarely HIFU, helpfulness in both pretreatment planning and real-time imaging during ablation. MRI is the excellent modality for guidance during ablation allowing high contrast visualization of the targeted lesions and surrounding organs. Real-time MRI thermometry information of the targeted tissue can be obtained during the treatment procedure, which will guarantee biological thermal effects at the focused point. Furthermore, real-time temperature monitoring attenuates the over-sonication risk and undesired damage to the adjacent organs. Finally, contrast-enhanced MRI was consumed to calculate non-vascularized volume of lesions reflected the nonviable tissue region [9-14].

\section{- Transurethral approach}

MRI-guided transurethral HIFU was carried out under general anesthesia accompanied by insertion of a suprapubic catheter and transurethrally inserted nitinol guidewire. The patient was located in supine position. An austere ultrasound supplicator consisted of a linear array of 10 liberated ultrasound transducer that diffuses directional highintensity sonication energy directly into the prostatic lesions was placed into the urethra over this guidewire. Computer-aided program was managed to image the targeted prostate margin during treatment planning, control the thermal energy delivery during HIFU ablation, and enforce the accurate temperature feedback algorithm (FIGURE 2). Under MRI guidance, the ultrasound supplicator was positioned exactly within the prostatic urethra with a 3 $\mathrm{mm}$ safety boundary between the ultrasound transducers and sphincter plane at the prostate apex. Then high-resolution prostate images were obtained for treatment planning. The clinicians monitor the outer prostate boundary cautiously. The target prostate lesions were sonicated over $55^{\circ} \mathrm{C}$ representative of thoroughly acute thermal coagulative necrosis under the real-time MRI thermometry guidance acquired every $6 s$, which generates continuous evaluation of a three-dimensional temperature volume during ablation. The suprapubic catheter was remained in place for two weeks. The patient will be discharged on the same day if without complications [9-12]. 


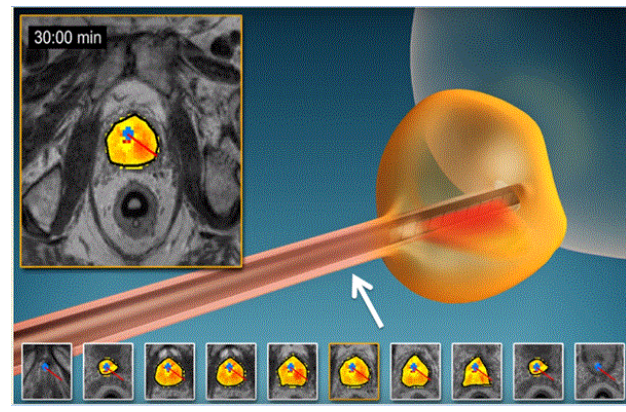

Figure 2. Magnetic resonance-guided HIFU with the transurethral approach (white arrow).

\section{- Transrectal approach}

MRI-guided transrectal HIFU was exploited 990 element phased-array HIFU endorectal transducer placed into the rectum and filled with degassed $14{ }^{\circ} \mathrm{C}$ water to not solely eliminate air within the beam path but also chill the rectum. The patients were located in a low lithotomy position on with insertion of a suprapubic catheter for constant bladder drainage during ablation. All patients were indicated general anesthesia with midazolam, fentanyl, and propofol. T2-weighted images were acquired to confine the targeted lesion and allow treatment planning. Urologists and interventional radiologists contour manually prostate, targeted lesion, urethra, anterior rectal wall, bladder apex, vesicles, and bundle nerves. The treatment software automatically generated the treatment plan and recommended the essential energy level at a frequency of $2.3 \mathrm{mHz}$ and power of $30 \mathrm{~W}$, the number of sonications, and the sonication areas' shape and size. Though the ultrasound transducer typically emits a power of $30 \mathrm{~W}$, this ultrasonic power can change along with the lesion location, tissue absorption, and accumulative regional heat. At the beginning of treatment, test of sonication for verifying was carried out to confirm targeted area. The focused sonication beam path was manifested over images of anatomy.

After verification, multiple sonications were initiated to consecutive targeted lesion inside the available region of treatment. In order to obtain homogeneous coagulative necrotic tumor, subsequent sonication overlaid with the previous sonication, and a $5 \mathrm{~mm}$ boundary surrounding the lesion were required to ablate. It is recommended that a $5 \mathrm{~mm}$ tumor-free margin was limited by clinician-defined vulnerable organs such as rectal wall, external urethral sphincter, bladder wall, neurovascular bundles, urethra which need to be conserved.
MRI thermography monitored procedure of ablation (FIGURE 3). HIFU treatment was regarded successfully when the lesion's temperature was at least $65^{\circ} \mathrm{C}$. When targeted lesion's temperature was not elevated up to the temperature purpose, additional sonication was needed $[13,14]$.

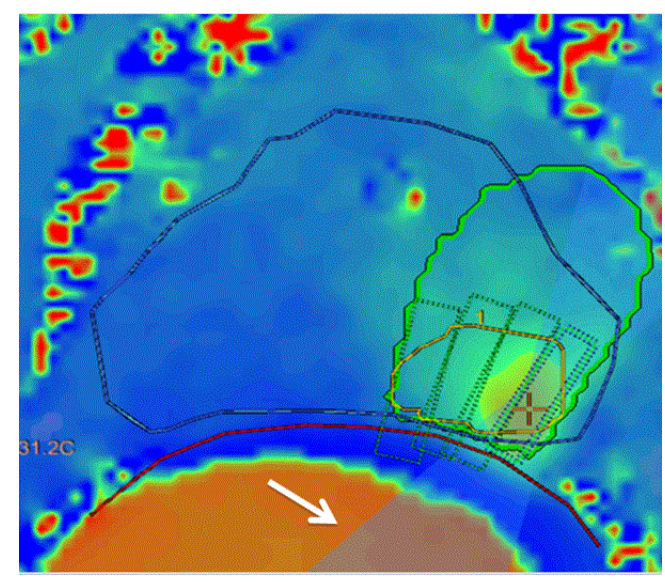

Figure 3. Magnetic resonance-guided HIFU with the transrectal approach (white arrow).

\section{- MRI guided HIFU Versus US guided HIFU}

The advantage of MRI-guided HIFU in comparison with US-guided HIFU is the capability of real-time thermometry, which improves precise therapeutic ablation by guaranteeing targeted lesion's temperature rise in conjunction with facilitating closed-loop feedback monitor. Furthermore, guidance of MRI was superior to guidance of ultrasound because of a better tissue contrast and large field of view owing to avoid sonicating outside the targeted lesions. Nonetheless, the main disadvantage of MRI-guided HIFU in comparison with ultrasound-guided HIFU is installed cost and maintenance cost. In addition, MRI-guided HIFU consumes more space than ultrasound-guided HIFU. MRIguided HIFU is more sensitive to motions: patient movements during ablation and bowel movements due to lower temporal resolution in collecting real-time data $[4,10-14]$.

\section{Complications}

There are some adverse events which can appear in the treatment of both BPH and prostate cancer such as bladder neck stenosis, urethral stricture stenosis, urinary tract infection, urinary incontinence, urinary retention, impotence, rectal perforation, rectourethral fistula, hematuria, epididymitis, rectal pain, fecal straining and pelvic bloating [6,7,11-15]. Furthermore, one of the most 
problematic conditions in HIFU for prostate cancer was the residual cancerous cells at the margin between ablated area and normal area led to the recurrent of disease. In order to avoid this severe circumstance, the screening multi-parametric MRI plays a crucial role on investigating precise prostate cancer's stage prior to the treatment [11-15].

\section{Conclusion}

HIFU is a novel non-invasive method for benign prostatic hyperplasia and prostate cancer. There are many approaches of HIFU under the guidance of MRI or ultrasound and the location of treatment transducer inside the rectum or urethra. Each approach for patients with benign prostatic hyperplasia and prostate cancer has advantage and disadvantage mainly based on the guided modalities; hence, further studies should be launched to validate and compare the efficacy and safety of these approaches.

\section{Disclosure statement}

Nguyen Minh Duc and Huynh Quang Huy contributed equally to this article. All authors read and approved manuscript. The authors of this manuscript report no conflict of interest.

\section{References}

1. Linares-Espinos E, Carneiro A, Martínez-Salamanca JI et al. New technologies and techniques for prostate cancer focal therapy: a review of the current literature. Minerva. Urol Nefrol. 70: 252-263, (2018).

2. Duran-Rivera A, Montoliu GA, Juan EJ et al. High-intensity focused ultrasound therapy for the treatment of prostate cancer: Medium-term experience. Actas. Urol. Esp. 42: 450-456, (2018).

3. Aoun F, Marcelis Q, Roumeguere T. Minimally invasive devices for treating lower urinary tract symptoms in benign prostate hyperplasia: technology update. Res. Rep. Urol. 7: 125-136, (2015).

4. Kim YS. Advances in MR image-guided high-intensity focused ultrasound therapy. Int. J. Hyperthermia. 31: 225-232, (2015).

5. Van Hest, D'Ancona F. Update in minimal invasive therapy in benign prostatic hyperplasia. Minerva. Urol. Nefrol. 61: 257-268, (2009).
6. Lu J, Hu W, Wang W. Sonablate-500 transrectal high-intensity focused ultrasound (HIFU) for benign prostatic hyperplasia patients. J. Huazhong. Univ. Sci. Technolog. Med. Sci. 27 671-674, (2007).

7. Madersbacher S, Schatzl G, Djavan B et al. Long-term outcome of transrectal high- intensity focused ultrasound therapy for benign prostatic hyperplasia. Eur. Urol. 37: 687-694, (2000).

8. Sankineni S, Wood BJ, Rais-Bahrami S et al. Image-guided focal therapy for prostate cancer. Diagn. Interv. Radiol. 20: 492-497, (2014).

9. Sommer G, Pauly KB, Holbrook A et al. Applicators for magnetic resonance guided ultrasonic ablation of benign prostatic hyperplasia. Invest. Radiol. 48: 387-394, (2013)

10. Burtnyk M, Hill T, Cadieux-Pitre $\mathrm{H}$ et al. Magnetic resonance image guided transurethral ultrasound prostate ablation: a preclinical safetyand feasibility study with 28-day followup. J. Urol. 193: 1669-1675, (2015).

11. Chin JL, Billia M, Relle J et al. Magnetic Resonance Imaging-Guided
Transurethral Ultrasound Ablation of Prostate Tissue in Patients with Localized Prostate Cancer: A Prospective Phase 1 Clinical Trial. Eur Urol. 70: 447-455, (2016).

12. Chopra R, Colquhoun A, Burtnyk M et al. MR imaging-controlled transurethral ultrasound therapy for conformal treatment of prostate tissue: initial feasibility in humans. Radiology. 265: 303-313, (2012).

13. Ghai S, Louis AS, Van Vliet M et al. Real-Time MRI Guided Focused Ultrasound for Focal Therapy of Locally Confined Low Risk Prostate Cancer: Feasibility and Preliminary Outcomes. AJR. Am. J. Roentgenol. 205: 177-184, (2015).

14. Ghai S, Perlis N, Lindner $U$ et al Magnetic resonance guided focused high frequency ultrasound ablation for focal therapy in prostate cancer - phase 1 trial. Eur. Radiol. 28: 4281-4287, (2018).

15. Zini C, Hipp E, Thomas S et al Ultrasound- and MR-guided focused ultrasound surgery for prostate cancer. World. J. Radiol. 4: 247-252, (2012). 